\title{
First UK dental mentoring conference is a success
}

The first conference specifically for mentoring in dentistry organised by the Dental Coaching Academy was held on 20 October 2018, hosted by the BDA in London, attended by more than 60 delegates.

The day began with a trio of excellent keynote speakers led by Professor David Clutterbuck, the internationally renowned authority on mentoring. Yvonne Coghill gave a deeply personal and UK health-service perspective and Dr Catherine Rutland gave the audience plenty to ponder from the private sector viewpoint.

Clutterbuck began the proceedings, taking a broad-brush approach to mentoring. Coghill narrowed the focus to healthcare and Rutland described how
Simplyhealth Professionals were applying mentoring in support of their practices and practitioners.

The three keynote speakers led the delegates in a wide-ranging panel discussion followed by an unexpected gift to delegates when Clutterbuck offered all delegates a copy of his work How to ask powerful questions.

Delegate feedback was excellent for all the keynote speakers, with comments such as 'Amazing context. Made me feel confident of taking up mentoring' and 'Inspiring lecture that taught me the importance of not giving up following knock backs.'

This session was followed by parallel streams led by three speakers well known to many participants - Alasdair Miller, Joanne Brindley and Sally Strazzanti.
They introduced delegates to 'Mindsets in Mentoring, 'What can Sinatra teach us?' and 'Mentoring for Resilience'.

Smaller group practical sessions were led by Pam Coates, Bob Hughes, Fiona Ellwood and Hoda Wassif. These session topics included 'Stepping from Associate to Practice owner,' 'Mentoring principles', 'Ethical practice and mentoring agreements' and 'Culture sensitive mentoring.

A mentoring café held at lunchtime with experienced mentors offering taster 15-minute mentoring sessions to delegates proved successful.

This year's conference will be held on 23 November 2019.

By Janine Brooks, Director of Dental Programmes for the Dental Coaching Academy

\section{Health benefits of sugar alternatives questioned in study}

Artificial sweeteners and sugar alternatives may not be as healthy as generally thought, according to a recent study ${ }^{1}$ published in the British Medical Journal.

Experts have found there was no compelling evidence to suggest any important health benefits from consuming non-sugar sweeteners and there could even be potential harms.

Worries over health and quality of life have encouraged many people to adopt healthier lifestyles and avoid foods rich in sugars, salt, or fat, so foods and drinks containing non-sugar sweeteners rather than regular sugars have become increasingly popular.

Several non-sugar sweeteners are approved for use but less is known about their potential benefits and harms within acceptable daily intakes because there is often only limited and conflicting evidence.

A team of European researchers, therefore, led by the University of Freiburg in Germany, set out to gain a better understanding of these potential benefits and harms by analysing 56 published studies comparing no intake or lower intake of non-sugar sweeteners with higher intake in healthy adults and children.

Measures included weight, glycaemic control, oral health, cancer, cardiovascular disease, kidney disease, mood and behaviour.
Overall, the results showed that for most outcomes, there appeared to be no statistically or clinically relevant differences between people exposed to non-sugar sweeteners and those not exposed, or between different doses of non-sugar sweeteners.

In adults, for example, the findings from a few small studies suggested small improvements in BMI (body mass index) and fasting blood glucose levels with non-sugar sweeteners, but the certainty of this evidence was low.

Lower intakes of non-sugar sweeteners were associated with slightly less weight gain than higher intakes, but the certainty of this evidence was also low.

The results showed that in children, a smaller increase in BMI score was seen with non-sugar sweeteners compared with sugar, but intake of non-sugar sweeteners made no differences to body weight.

There was no good evidence of any effect of non-sugar sweeteners found for overweight or obese adults or children actively trying to lose weight.

The researchers described their study as the most comprehensive review on this topic to date and said it would inform a World Health Organisation guideline for health experts and policy makers.

They stressed that the quality of evidence in many of the studies was low, so confidence in the results was limited, adding that longer-term studies were needed to clarify whether non-sugar sweeteners were a safe and effective alternative to sugar.

They concluded: 'There was no compelling evidence to indicate important health benefits of non-sugar sweetener use on a range of health outcomes. Potential harms from the consumption of non-sugar sweeteners could not be excluded.'

A BDA spokesperson said: 'We don't recommend any 'sugar-free' alternatives to fizzy drinks other than milk and water. We remain concerned that many soft drinks either encourage a sweet tooth, or come with high levels of acidity that harm oral health.

'Every Christmas, Coca Cola spin doctors try their best to claim there's a "healthy option" for teeth while marketing low or no sugar products more acidic than vinegar or lemon juice. When nearly half of teenagers are showing signs of dental erosion dentists know many of these brands have as little place as a festive tradition as their sugarladen stablemates.'

1. Toews I, Lohner S, Küllenberg de Gaudry D, Sommer $\mathrm{H}$, and Meerpohl J J. Association between intake of non-sugar sweeteners and health outcomes: systematic review and meta-analyses of randomised and non-randomised controlled trials and observational studies. BMJ 2018; 363: k4718. DOI: 10.1136/bmj.k4718. 